Running head: host nutrition and virus interactions

\title{
1 Host nutrition mediates interactions between plant viruses, altering transmission and
}

\section{2 predicted disease spread}

4 Amy E. Kendig (aekendig@gmail.com)1,2, Elizabeth T. Borer (borer@umn.edu) ${ }^{1}$, Emily N.

5 Boak (boakn009@tamu.edu) $)^{1,3}$, Tashina C. Picard (picar022@umn.edu) ${ }^{1}$, and Eric W. Seabloom

6 (seabloom@umn.edu $)^{1}$

8 Author affiliations:

9 1. Department of Ecology, Evolution, and Behavior, University of Minnesota, St. Paul, MN

1055108, USA.

11 2. Present address: Agronomy Department, University of Florida, Gainesville, FL 32611, USA.

12 3. Present address: Department of Horticultural Sciences, Texas A\&M University, College

13 Station, TX 77843, USA.

14

15 Corresponding author: Amy Kendig, aekendig@gmail.com 


\section{Abstract}

Interactions among co-infecting pathogens are common across host taxa and can affect infectious disease dynamics. Host nutrition can mediate these among-pathogen interactions, altering the establishment and growth of pathogens within hosts. It is unclear, however, how nutrition-

21 mediated among-pathogen interactions affect transmission and the spread of disease through

22 populations. We manipulated the nitrogen $(\mathrm{N})$ and phosphorus $(\mathrm{P})$ supplies to oat plants in

23 growth chambers and evaluated interactions between two aphid-vectored Barley and Cereal

24 Yellow Dwarf Viruses: PAV and RPV. We quantified the effect of each virus on the other's establishment, within-plant density, and transmission. Co-inoculation significantly increased PAV density when $\mathrm{N}$ and P supplies were low and tended to increase RPV density when N supply was high. Co-infection increased PAV transmission when $\mathrm{N}$ and $\mathrm{P}$ supplies were low and tended to increase RPV transmission when N supply was high. Despite the parallels between the effects of among-pathogen interactions on density and transmission, changes in virus density only partially explained changes in transmission, suggesting that virus density-independent

31 processes contribute to transmission. A mathematical model describing the spread of two viruses through a plant population, parameterized with empirically derived transmission values,

33 demonstrated that nutrition-mediated among-pathogen interactions could affect disease spread.

34 Interactions that altered transmission through virus density-independent processes determined

35 overall disease dynamics. Our work suggests that host nutrition alters disease spread through

36 among-pathogen interactions that modify transmission.

37 Key words: co-infection, transmission, within-host, disease spread, barley and cereal yellow

38 dwarf viruses, Avena sativa, nitrogen, phosphorus, Rhopalosiphum padi 


\section{Introduction}

Resource supply can alter the outcome of species interactions (Tilman 1977, Maestre and

42 Cortina 2004). A rich body of theoretical and empirical literature has explored the effects of

43 resource supply on ecological dynamics, but most has focused on free-living organisms (Bruno

44 et al. 2003, Miller et al. 2005). The nutrients consumed by hosts (i.e., host nutrition) are

45 important mediators of resource supply to assemblages of pathogens and other symbionts (Smith

46 et al. 2005). Host nutrition can affect interactions among pathogens that co-infect plant or animal

47 hosts (Lacroix et al. 2014, Lange et al. 2014, Budischak et al. 2015, Wale et al. 2017) -

48 interactions that can influence host survival, transmission between hosts, and evolution of

49 virulence (Vasco et al. 2007, Tollenaere et al. 2016). Therefore, host nutrition may affect

50 infectious disease dynamics by altering among-pathogen interactions.

Among-pathogen interactions can have positive, neutral, or negative effects on within-

52 host pathogen fitness (Moreno and López-Moya 2020). Competition among pathogens for

53 limiting resources, such as nutrients, cells, or tissues, can suppress pathogen densities (Smith and

54 Holt 1996, Pedersen and Fenton 2007). Host nutrition that affects the supply of pathogen-

55 limiting resources can alter the outcome of pathogen competition (Wale et al. 2017). Pathogens

56 also can interact indirectly by promoting or suppressing host immune reactions (Pedersen and

57 Fenton 2007, Vasco et al. 2007). Immune functioning in mammals depends on vitamins, zinc,

58 iron, and iodine (Katona and Katona-Apte 2008), and plant susceptibility to infection can depend

59 on nitrogen $(\mathrm{N})$, phosphorus $(\mathrm{P})$, and potassium $(\mathrm{K})$ in the soil (Dordas 2009). It follows that host

60 nutrition also can affect immune-mediated pathogen interactions (Budischak et al. 2015).

61 Interactions among pathogens can affect disease spread when there is a strong

62 relationship between within-host pathogen density and processes that affect host population 
63 dynamics, including transmission, mortality, and recovery (Mideo et al. 2008, Handel and

64 Rohani 2015). Pathogens that reach higher densities within hosts are more likely to produce

65 more propagules for transmission (McCallum et al. 2017). Interactions among pathogens within

66 plants and animals alter transmission and the proportion of the population that becomes infected

67 (Ezenwa and Jolles 2011, Susi et al. 2015a, Halliday et al. 2017). Yet, it is unclear how the

68 impacts of host nutrition on among-pathogen interactions affect disease spread. Nutrition-

69 mediated interactions within the host are likely to influence disease spread if a strong

70 relationship between within-host pathogen density and a process that affects host population

71 dynamics (e.g., transmission) exists (Gilchrist and Coombs 2006, Strauss et al. 2019).

Increases in within-host pathogen densities do not always increase the probability of

73 transmission (Handel and Rohani 2015, McCallum et al. 2017). For example, the relationship

74 between pathogen density and transmission is sigmoidal for malaria-inducing Plasmodium

75 falciparum, and increases in $P$. falciparum density beyond a threshold do not affect transmission

76 (Alizon and van Baalen 2008). Such non-linearities can arise when vector behavior and

77 pathogen-vector interactions affect the probability of transmission (Gray et al. 1991), decoupling

78 transmission from within-host dynamics. Interactions among pathogens also can change

79 establishment or transmission independently of changes in pathogen density. For example, co-

80 infection can modify vector preference and the efficacy of vector transmission, causing

81 transmission from co-infected hosts to differ from singly infected hosts (Rochow et al. 1983,

82 Srinivasan and Alvarez 2007). Therefore, nutrition-mediated among-pathogen interactions may

83 modify disease spread through processes that are independent of within-host density. 
crops and wild plants: the Barley and Cereal Yellow Dwarf Viruses (B/CYDVs; Power et al.

2011). In a growth chamber experiment, we manipulated soil $\mathrm{N}$ and $\mathrm{P}$ concentrations supplied to oat plants singly- and co-inoculated with two B/CYDVs: BYDV-PAV (PAV, hereafter) and

CYDV-RPV (RPV, hereafter). We quantified the effects of interactions between the viruses by

measuring establishment, within-plant virus densities, and transmission to new plants (Fig. 1).

91 Then, we used empirically estimated transmission values to parameterize a mathematical model

with the goal of predicting the effects of host nutrition-mediated among-pathogen interactions on

disease spread. We used this experiment and model to address the following questions.

Question 1: Does host nutrition affect among-pathogen interactions within hosts?

Results from previous studies indicate that host nutrition mediates B/CYDV replication

and among-pathogen interactions (Lacroix et al. 2014, Whitaker et al. 2015). However, it is

103 evaluate how host nutrition affected the nature (i.e., positive, neutral, negative) and timing (i.e.,

104 early or late relative to inoculation) of interactions between B/CYDVs within plants. While this

105 experimental design allowed us to test whether co-inoculation altered establishment or post-

106 establishment processes, it did not allow us to fully discern the mechanisms behind observed

107 among-pathogen interactions (e.g., resource competition, immune-mediated interactions).

108 Question 2: Does host nutrition modify among-pathogen interactions to affect transmission? 
111 density are expected to promote (suppress) transmission. Virus density-transmission

112 relationships, however, may be virus-specific (Gray et al. 1991). In addition, the impacts of co-

113 infection on vector acquisition may affect transmission independently of virus density (Rochow

114 et al. 1983, Wen and Lister 1991). We evaluated the effects of virus density, host nutrition, and

115 co-infection on transmission from source plants (i.e., those inoculated in Question 1) to recipient

116 plants grown with a full factorial combination of low and high $\mathrm{N}$ and $\mathrm{P}$ supply rates.

119 changes in transmission due to host nutrition-mediated among-pathogen interactions are

120 expected to have population-level consequences. Nutrient additions have altered B/CYDV

121 infection prevalence in wild grass populations (Seabloom et al. 2013, Borer et al. 2014), but it is

122 unclear whether these changes were mediated by within-plant dynamics. We parameterized a

123 two-pathogen compartmental model with our empirical results to quantify the effects of

124 nutrition-mediated among-pathogen interactions on infection prevalence over time. We then used

125 the model to evaluate the contribution of within-plant virus density to disease dynamics.

\section{Methods}

The B/CYDV group consists of single-stranded RNA viruses in the Luteoviridae family

129 that can infect over 100 species of Poaceae and are persistently transmitted by several aphid

130 species (Power et al. 2011). The aphid Rhopalosiphum padi is an effective vector of the two

131 virus species we used in this study, PAV and RPV (Gray et al. 1991, Power et al. 2011). We 
132 maintained cultures of PAV and RPV in Avena sativa L. cv. Coast Black Oat (National plant

133 germplasm system, USDA) by periodically feeding infected plant tissue to $R$. padi aphids, which

134 were temporarily transferred to uninfected $A$. sativa. Rhopalosiphum padi colonies were

135 maintained on uninfected A. sativa plants. We obtained the virus isolates from Dr. Stewart Gray

136 at Cornell University (Ithaca, NY, USA), and the aphids from Dr. George Heimpel at the

137 University of Minnesota (St. Paul, MN, USA), who each collected these organisms in their

138 respective states. Uninfected plants, infected plants, and plants with $R$. padi were grown in

139 Sunshine MVP potting soil (Sun Gro Horticulture, Agawam, MA, USA) and kept in separate

140 growth chambers at $20^{\circ} \mathrm{C}$ with a $16: 8 \mathrm{~h}$ light:dark cycle for one year prior to the experiment.

The experiment was carried out from February to August 2014 over five temporal blocks.

143 Avena sativa seeds were germinated in $164 \mathrm{~mL}$ conical pots with $70 \%$ Sunshine medium

144 vermiculite (vermiculite and $<1 \%$ crystalline silica; Sun Gro Horticulture) and 30\% Turface

145 MVP (calcined clay containing up to $30 \%$ crystalline silica; Turface Athletics, Buffalo Grove,

146 IL, USA) that had been saturated with tap water. Beginning two days after planting, we watered

147 each plant with one of the following modified Hoagland solutions (i.e., nutrient treatments,

148 Appendix S1: Table S1, Hoagland and Arnon 1938): $7.5 \mu \mathrm{M} N$ and $1 \mu \mathrm{M}$ P ("Low"), $7.5 \mu \mathrm{M} N$

149 and $50 \mu \mathrm{M}$ P ("P"), $375 \mu \mathrm{M} N$ and $1 \mu \mathrm{M}$ P ("N"), or $375 \mu \mathrm{M} N$ and $50 \mu \mathrm{M} P$ ("N+P"), which

150 differentially affect plant growth and B/CYDV infection prevalence (Seabloom et al. 2011,

151 Lacroix et al. 2014, 2017). Plants were watered with $30 \mathrm{~mL}$ of nutrient solution twice per week

152 prior to inoculation and weekly following inoculation. After inoculation, plants were moved to a

153 growth chamber maintained at $20^{\circ} \mathrm{C}$ with a $16: 8 \mathrm{~h}$ light:dark cycle under $28 \mathrm{~W}$ bulbs. 
15510 to 11 days post planting with PAV, RPV, or both (Fig. 1a). Aphids fed on virus culture leaves

156 for approximately two days and then were combined into plastic containers by inoculation type.

157 We attached one mesh cage to each plant on the largest leaf and placed ten aphids in each mesh

158 cage, allowing them to feed on the plants for approximately four days. PAV inoculations

159 involved five aphids that fed on PAV-inoculated culture leaves and five that fed on uninfected

160 leaves, RPV inoculations involved five aphids that fed on RPV-inoculated culture leaves and five

161 that fed on uninfected leaves, co-inoculations involved five aphids that fed on PAV-inoculated

162 culture leaves and five that fed on RPV-inoculated culture leaves (Appendix S1).

163 We destructively harvested the experimental plants at eight days post inoculation (DPI):

$1645,8,12,16,19,22,26$, or 29 days. We cut the stems and leaves into small pieces using a

165 sterilized blade, weighed them, stored about $60 \%$ of the tissue at $-80^{\circ} \mathrm{C}$ for reverse transcription-

166 quantitative polymerase chain reaction (RT-qPCR, see Quantifying virus density), and used the

167 remainder to measure transmission (Fig. 1b). Unique combinations of nutrient treatments $(n=4)$,

168 inoculation treatments $(n=3)$, and harvesting days $(n=8)$ resulted in 96 treatments. Each

169 treatment was replicated twice in block one, once in blocks two through four, and zero to two

170 times in block five, depending on losses in earlier blocks (Appendix S1: Table S2).

171 During blocks $1-4$, approximately $40 \%$ of the tissue from the Question 1 plants (i.e.,

172 "source plants") was used to measure transmission to four "recipient plants" grown in each of the

173 four nutrient treatments (Question 2, Fig. 1d). Source plant tissue was placed in glass tubes with

17425 aphids for about two days. Then, five aphids, contained in a mesh cage affixed to the largest

175 leaf of each recipient plant, fed for about four days (Appendix S1). The recipient plants were

176 harvested 14 to $15 \mathrm{DPI}$ and all of the stem and leaf tissue was stored at $-80^{\circ} \mathrm{C}$ for RT-PCR and

177 gel electrophoresis, which detects whether plants were infected with either virus (Appendix S1). 
To quantify virus densities — number of viruses per milligram plant —in source plants, we

180 first extracted the total RNA from $\sim 50 \mathrm{mg}$ of thawed plant tissue (Appendix S1, Fig. 1c). We

181 used one-step RT-qPCR to obtain the concentration of genomic RNA copies per volume of total

182 RNA extract (Appendix S1). The RNA regions targeted for RT-qPCR, which are specific to

183 PAV and RPV, encode coat proteins (Appendix S1: Table S3). We assumed that the genomic

184 RNA copies measured by RT-qPCR approximated the number of virus particles in a sample and

185 used the total amount of plant tissue extracted to estimate the concentration of viruses in $1 \mathrm{mg}$ of

186 plant tissue (Mackay et al. 2002, Lacroix et al. 2017). Virus densities that were large enough to

187 be quantified by RT-qPCR were considered indicators of virus establishment. Plants with

188 unintended infections (i.e., PAV detected in RPV-inoculated plants and RPV detected in PAV-

189 inoculated plants, Appendix S1: Table S2) were excluded from analyses to generate conservative

190 estimates. Such infections may have been caused by aphids that escaped inoculation cages or a

191 small number of unintended infections in the virus culture leaves (Appendix S1).

We performed all statistical analyses in R version 3.5.2 (R Core Team 2018), using the

194 brms package (Bürkner 2017) to fit Bayesian linear regressions to data for each virus species. To

195 evaluate virus establishment (Question 1), we fit a generalized linear regression with a Bernoulli

196 response distribution (logit-link) to the proportion of plants that tested positive for infection

197 based on RT-qPCR. To evaluate virus density in plants with infection (Question 1), we fit a

198 normal linear regression to log-transformed virus density. In both cases, the predictor variables

199 were a three-way interaction among the binary variables co-inoculation, $\mathrm{N}$ addition, and $\mathrm{P}$

200 addition (Appendix S2: Table S1). A first-order autocorrelation structure was used to account for 
201 correlations between virus density values on consecutive harvesting days. In the virus

202 establishment models, harvesting day was included as a random intercept, which accounts for

203 variation among harvesting days (autocorrelation structures were not compatible with this type of

204 model). Experimental block was not included as a random intercept in either model because it

205 explained minimal variation. We evaluated transmission (proportion of recipient plants infected,

206 Question 2) using a generalized linear model with a Bernoulli response distribution (logit-link):

$$
\begin{gathered}
\text { transmission } \sim \text { virus density } \times\left(N_{\text {source }} \times P_{\text {source }}+N_{\text {recipient }} \times P_{\text {recipient }}\right)+ \\
\text { coinfection } \times\left(N_{\text {source }} \times P_{\text {source }}+N_{\text {recipient }} \times P_{\text {recipient }}\right)
\end{gathered}
$$

208 Subscripts indicate which plant the nutrient treatment was applied to and virus density was

209 centered and scaled. Redundant main effects and interactions were omitted. Note that "co-

210 infection" describes the status of the plant while "co-inoculation" describes the experimental

211 treatment. Harvesting day and experimental block were included as crossed random intercepts.

212 We used data from an experiment that measured PAV and RPV densities and transmission under

213 similar conditions to inform some of the priors for the density and transmission models (Lacroix

214 et al. 2017); uninformative priors were used otherwise (Appendix S2: Table S1). All models

215 were run with three Markov chains, 6000 iterations each with a 1000 iteration warm-up. We

216 evaluated model fit with r-hat values and visual comparisons of the observed data and simulated

217 data from the posterior predictive distributions. We present the estimated effect sizes from

218 models with informative priors, which were similar to models without informative priors

219 (Appendix S2: Fig. S1). We report results as statistically significant if the $95 \%$ credible interval

220 (CI; the interval that contains the most probable estimate values) omits the value representing

221 "no effect" (i.e., zero for normal distribution or one for Bernoulli distribution).

223 To evaluate the effects of nutrition-mediated among-pathogen interactions on the spread 
of B/CYDVs in plant populations, we used our empirical results to parameterize a two-pathogen compartmental model (Seabloom et al. 2015). In the model, host plants are susceptible $(S)$, infected with PAV $\left(I_{P}\right)$, infected with RPV $\left(I_{R}\right)$, or co-infected $\left(I_{C}\right)$ :

$$
\begin{gathered}
\frac{d S}{d t}=-\left[\beta_{P} I_{P}+\beta_{R} I_{R}+q_{P} \beta_{P}\left(1-q_{R} \beta_{R}\right) I_{C}+q_{R} \beta_{R}\left(1-q_{P} \beta_{P}\right) I_{C}+q_{P} \beta_{P} q_{R} \beta_{R} I_{C}\right] \frac{S}{N} \\
\frac{d I_{P}}{d t}=\beta_{P}\left[I_{P}+q_{P}\left(1-q_{R} \beta_{R}\right) I_{C}\right] \frac{S}{N}-\beta_{R}\left(I_{R}+q_{R} I_{C}\right) \frac{I_{P}}{N} \\
\frac{d I_{R}}{d t}=\beta_{R}\left[I_{R}+q_{R}\left(1-q_{P} \beta_{P}\right) I_{C}\right] \frac{S}{N}-\beta_{P}\left(I_{P}+q_{P} I_{C}\right) \frac{I_{R}}{N} \\
\frac{d I_{C}}{d t}=\beta_{P}\left(I_{P}+q_{P} I_{C}\right) \frac{I_{R}}{N}+\beta_{R}\left(I_{R}+q_{R} I_{C}\right) \frac{I_{P}}{N}+q_{P} \beta_{P} q_{R} \beta_{R} I_{C} \frac{S}{N} \\
N(t)=S(t)+I_{P}(t)+I_{R}(t)+I_{C}(t)
\end{gathered}
$$

232 The terms $\beta_{P}$ and $\beta_{R}$ represent the probability of transmission from plants singly infected with

233 PAV and RPV, respectively, given vector-assisted contact with another plant. Transmission from 234 co-infected plants equals transmission from singly infected plants multiplied by a modifier $\left(q_{P}\right.$ or $\left.235 q_{R}\right)$, which may represent positive $(>1)$ or negative $(<1)$ interactions (Appendix S3). We 236 performed simulations of Eq. 2 over a single growing season (R version 3.5.2, R Core Team 237 2018) using the deSolve package (Soetaert et al. 2010). We compared simulations with both 238 viruses present in the system to those with each virus alone. We repeated the simulations with 239 three sets of parameter values estimated from Eq. 1 (Appendix S3: Table S1) that differ in the 240 processes by which virus interactions can affect transmission: through changes in virus density, 241 virus density-independent processes, and both types of processes (Appendix S3: Table S2).

242 Parameter values for each nutrient treatment were used in separate simulations, restricting 243 transmission to plants grown with the same nutrient treatment. 


\section{Results}

Question 1: Does host nutrition affect among-pathogen interactions within hosts?

Nutrient addition and co-inoculation did not significantly affect PAV or RPV

248 establishment (the proportion of plants infected; Appendix S2: Table S1). Co-inoculation had the

249 strongest effects on PAV establishment when plants were grown with low nutrients (-29\%, Fig.

250 2c) and RPV establishment when plants were grown with elevated $\mathrm{N}(+7 \%$, Fig. 2d).

Nutrient addition and co-inoculation did not significantly affect RPV density (viruses per

252 mg plant tissue; Table 1). Co-inoculation had the strongest effect on RPV density when plants

253 were grown with elevated N (+105\%, Fig. 2 h). This positive effect was relatively consistent

254 following the first two harvesting days (Fig. 2f). Co-inoculation significantly increased PAV

255 density 98\% when plants were grown with low nutrients (Fig. 2g, Table 1), which was more

256 evident later in the course of infection (Fig. 2e).

257 Question 2: Does host nutrition modify among-pathogen interactions to affect transmission?

258 Host nutrition modified the relationships between virus density and transmission

259 (proportion of recipient plants infected; Table 2). RPV density significantly increased

260 transmission when recipient plants were grown with elevated $\mathrm{N}$ and $\mathrm{P}$ (Fig. 3d). PAV displayed a

261 similar trend (Fig. 3c). PAV density significantly decreased transmission when recipient plants

262 were grown with elevated P (Fig. 3c); a trend also observed for RPV (Fig. 3d).

263 Consistent with the results for PAV density (Fig. 2g), co-infection significantly increased

264 PAV transmission $43 \%$ when source plants were grown with low nutrients and recipient plants

265 were grown with elevated P (Fig. 3e, Table 2). However, PAV density reduced transmission

266 under these conditions (Fig. 3c) and co-infection increased PAV transmission 93\% when density

267 was held constant (i.e., the difference in transmission at the vertical dotted line on Fig. 3c). Co- 
infection significantly reduced PAV transmission $26 \%$ from plants grown with elevated $\mathrm{N}$ to plants grown with low nutrients, with stronger effects when density was held constant (-38\%,

Fig. 3e). Nitrogen addition to recipient plants significantly increased RPV transmission 26\%

271 (source plants grown with low nutrients; Fig. 3f). Co-infection did not significantly affect RPV

272 transmission, increasing it the most when source plants were grown with elevated $\mathrm{N}$ and

273 recipient plants were grown with low nutrients (14\%, Fig. 3f). This result is consistent with the

274 positive effect of co-inoculation on RPV density (Fig. 2h), but co-infection still increased

275 transmission 14\% when density was held constant.

Question 3: Can nutrition-mediated among-pathogen interactions affect disease spread? infection prevalence in plant populations grown with elevated $\mathrm{P}$ and decrease PAV prevalence with the addition of $\mathrm{N}$ or both nutrients (Fig. 4a). These effects are driven by among-pathogen

280 interactions that do not act on transmission through changes in virus density (i.e., density-

281 independent, Fig. 4c). In fact, interactions with RPV that alter PAV density increase PAV

282 infection prevalence with $\mathrm{N}$ addition (Fig. 4b). Simulations suggest that PAV can increase RPV

283 infection prevalence with $\mathrm{N}$ addition and decrease RPV infection prevalence when plants are

284 grown with low nutrients (Fig. 4d). Again, these effects are driven by virus density-independent

285 processes (Fig. 4f) and changes in density due to among-pathogen interactions have some

286 opposite effects (Fig. 4e). The predicted effects begin about midway through the growing season 287 and later decline as all plants in the population become infected (Appendix S3: Fig. S1).

\section{Discussion}

The results from this experiment are consistent with findings from previous studies across 
291 2014, Lange et al. 2014, Budischak et al. 2015, Wale et al. 2017). We built upon this work to

292 demonstrate that host nutrition and among-pathogen interactions can alter transmission, and

293 potentially disease spread. In particular, plant viruses can promote one another's within-host

294 densities under specific host nutrition conditions (Question 1). The viruses had positive,

295 negative, and neutral effects on one another's transmission, which also varied with host nutrition

296 (Question 2). A mathematical model parameterized with these experimental results suggests that

297 interactions between viruses that alter transmission directly_as opposed to indirectly through

298 changes in density — will affect disease spread in a population (Question 3).

The effects of host nutrition on among-pathogen interactions within the host

Changes in host nutrition shifted among-pathogen interactions from neutral to positive.

301 With low nutrients, co-inoculation slowed the establishment of PAV, but ultimately promoted

302 PAV density. In contrast, co-inoculation had limited effects on PAV with elevated N and P

303 supplies. Co-inoculation increased RPV density with $\mathrm{N}$ addition. These results suggest that $\mathrm{N}$

304 and $\mathrm{P}$ supplied to grasses through fertilization, atmospheric deposition, and other processes, may

305 alter the strength of interactions between viruses co-occurring within hosts. We used previous

306 studies on nutrition-mediated PAV and RPV interactions to inform the priors of our statistical

307 models. In the previous studies, elevated $\mathrm{N}$ alleviated a negative effect of co-inoculation on RPV

308 establishment, but host nutrition did not mediate the effects of co-inoculation on virus density

309 (Lacroix et al. 2014, 2017). We also found that $\mathrm{N}$ addition led to a more positive effect of co-

310 inoculation on RPV. Our result that co-inoculation increased PAV density with low nutrient

311 supply provided a novel insight into our understanding of among-pathogen interactions.

312 Our work is consistent with previous studies that have shown that B/CYDVs, like other

313 plant viruses, can have positive effects on one another. Although it is not yet known how PAV 
314 and RPV apparently facilitate each other, there are at least two potential explanations. Plant

315 viruses can use proteins produced by other virus species, which may facilitate transmission and

316 movement through the plant (Wen and Lister 1991, Moreno and López-Moya 2020). Different

317 plant viruses also can interfere with host immunity using distinct mechanisms (Liu et al. 2012).

318 Complementarity in host immunosuppression may increase virus density (Moreno and López-

319 Moya 2020). Both mechanisms — sharing resources and complementary immunosuppression-

320 may be mediated by host nutrition (Smith et al. 2005, Budischak et al. 2015).

The effects of nutrition-mediated among-pathogen interactions on transmission

Host nutrition mediated the size and direction of the effects of among-pathogen

323 interactions on transmission. Although the positive effects of co-infection on transmission

324 occurred under the same nutrient treatments as positive effects of co-inoculation on density,

325 higher virus density did not explain increased transmission. In fact, the relationship between

326 virus density and transmission was variable for both viruses. This results is similar to a field

327 experiment manipulating plant fungal infection in which plants with more infected leaves did not

328 consistently produce more fungal spores (Susi et al. 2015b). Also, the density-transmission

329 relationship depended upon host nutrition, a result that is parallel to nutrition effects on aphid

330 endosymbionts (Wilkinson et al. 2007), suggesting that these interdependencies may be general.

331 A range of factors other than within-host pathogen density can affect transmission

332 (McCallum et al. 2017). B/CYDV transmission depends on virus-vector and host-vector

333 interactions (Rochow et al. 1983, Gray et al. 1991, Wen and Lister 1991). Highly relevant to our

334 results are the findings that plant nutrient content and infection status affect aphid feeding

335 preferences (Srinivasan and Alvarez 2007, Nowak and Komor 2010) and that the time aphids

336 spend feeding on plants can affect transmission (Gray et al. 1991). While we partially controlled 
337 for aphid preference by placing aphids in cages, we do not know how long aphids fed on each

338 plant. Thus, observed changes in transmission due to host nutrition and co-infection may have

339 arisen through variation in aphid feeding times. In this case, the presence of one pathogen can

340 affect the fitness and prevalence of the other (i.e., an "among-pathogen interaction"), despite the

341 absence of relevant changes in within-host density.

342 The implications of host nutrition-mediated among-pathogen interactions for disease spread

343 A mathematical model parameterized with empirical transmission values demonstrated

344 that nutrition-mediated among-pathogen interactions may affect infection prevalence in plant

345 populations and highlighted the importance of virus density-independent processes in

346 transmission. The result that changes in within-host virus density due to nutrition-mediated

347 among-pathogen interactions are unlikely to affect infectious disease dynamics in host

348 populations is consistent with research in animal populations demonstrating that nutrients can

349 influence infection prevalence through transmission processes that are independent of within-

350 host pathogen density, such as contact between susceptible and infectious hosts (Becker et al.

351 2015). Evaluating the relative effects of within-host dynamics and other transmission-related

352 processes on infectious disease dynamics is an important goal of disease ecology, especially

353 considering the complexity that within-host dynamics can add to empirical and theoretical

354 studies (Mideo et al. 2008, Handel and Rohani 2015, Susi et al. 2015b).

355 Nonetheless, some of the predictions of the model were not apparently consistent with

356 previous work. In two separate field experiments, $\mathrm{P}$ addition, but not $\mathrm{N}$ addition, increased PAV

357 prevalence, and in one experiment, neither nutrient affected RPV prevalence (Seabloom et al.

358 2013, Borer et al. 2014). Our model predicted that P addition would reduce PAV prevalence,

359 despite positive effects of co-infection under this condition. Both $\mathrm{N}$ and $\mathrm{P}$ were predicted to 
360 increase RPV prevalence. The effects of co-infection and host nutrition on aphid preference,

361 aphid population growth, and other factors affecting transmission that were not measured in this

362 experiment may explain the gap between model predictions and field experiment results.

363 Experiments examining such processes (e.g., Srinivasan and Alvarez 2007, Nowak and Komor

364 2010) could be paired with more detailed models (e.g., Strauss et al. 2019) to further explore the

365 implications of nutrition-mediated among-pathogen interactions for infectious disease dynamics.

\section{Limitations of this study}

We observed high uncertainty around estimates of establishment, pathogen density, and transmission, which may result from variation in host-pathogen interactions across individuals

369 (de Roode et al. 2004). This variation could be amplified in our dataset if it is more apparent

370 when viruses reach higher densities: the lower detection threshold of our RT-qPCR protocol

371 (about 150 viruses per mg plant) limited our ability to accurately quantify samples with low virus

372 densities, leading to their removal from density and transmission analyses. In addition, we

373 conducted simultaneous inoculations of PAV and RPV, but the sequence and timing of

374 inoculations can affect the outcome of pathogen interactions (Clay et al. 2018). Host nutrition

375 may have different effects on pathogen interactions depending on inoculation sequence and

376 timing. Nonetheless, our results do empirically demonstrate that a host's nutritional environment

377 can alter among-pathogen interactions, transmission, and disease spread.

381 manifested as changes in transmission that were independent of within-host pathogen density.

382 Therefore, the development of a more comprehensive, predictive framework for the role of co- 
383 infection in disease transmission and infectious dynamics would benefit from investigations of

384 host nutrition effects on virus-vector and host-vector interactions (Rochow et al. 1983, Nowak

385 and Komor 2010). Co-infection of hosts is common in natural systems (Tollenaere et al. 2016),

386 where host nutrition is altered by intentional and unintentional nutrient inputs (Smith et al. 2005).

387 Overall, the results from this study suggest that nutrient inputs into terrestrial plant systems are

388 likely to affect interactions between co-occurring viruses, leading to shifts in disease spread.

\section{Acknowledgements}

We are grateful to Christelle Lacroix, Melissa Rudeen, Anita Krause, Nicholas Cupery,

392 and to multiple anonymous reviewers for their comments on earlier drafts. AEK was supported

393 by an NSF IGERT graduate fellowship at the University of Minnesota (DGE-0653827) and an

394 NSF Graduate Research Fellowship (base award number 006595) and ETB and EWS received

395 support from the NSF program in Ecology and Evolution of Infectious Diseases (grant DEB-

396 1015805). AEK, ETB, and EWS designed the experiment, AEK, ENB, and TCP performed the

397 experiment and analyses, AEK wrote the first draft, and all authors contributed to revisions.

\section{Literature cited}

399 Alizon, S., and M. van Baalen. 2008. Transmission-virulence trade-offs in vector-borne diseases.

$400 \quad$ Theoretical Population Biology 74:6-15.

401 Becker, D. J., D. G. Streicker, and S. Altizer. 2015. Linking anthropogenic resources to wildlife402 pathogen dynamics: A review and meta-analysis. Ecology Letters 18:483-495.

403 Borer, E. T., E. W. Seabloom, C. E. Mitchell, and J. P. Cronin. 2014. Multiple nutrients and

404 herbivores interact to govern diversity, productivity, composition, and infection in a $405 \quad$ successional grassland. Oikos 123:214-224. 
Bruno, J. F., J. J. Stachowicz, and M. D. Bertness. 2003. Inclusion of facilitation into ecological theory. Trends in Ecology and Evolution 18:119-125.

Budischak, S. A., K. Sakamoto, L. C. Megow, K. R. Cummings, J. F. Urban, and V. O. Ezenwa. 2015. Resource limitation alters the consequences of co-infection for both hosts and parasites. International Journal for Parasitology 45:455-463.

Bürkner, P.-C. 2017. brms: An R package for Bayesian multilevel models using Stan. Journal of Statistical Software 80:1-28.

Clay, P. A., K. Dhir, V. H. W. Rudolf, and M. A. Duffy. 2018. Within-host priority effects systematically alter pathogen coexistence. The American Naturalist 193:187-199.

Dordas, C. 2009. Role of Nutrients in Controlling Plant Diseases in Sustainable Agriculture: A Review. Pages 443-460 in E. Lichtfouse, M. Navarrete, P. Debaeke, S. Véronique, and C. Alberola, editors. Sustainable Agriculture. Springer, Dordrecht, Netherlands.

Froissart, R., J. Doumayrou, F. Vuillaume, S. Alizon, and Y. Michalakis. 2010. The virulence-

Ezenwa, V. O., and A. E. Jolles. 2011. From host immunity to pathogen invasion: The effects of

Gilchrist, M. A., and D. Coombs. 2006. Evolution of virulence: interdependence, constraints, and helminth coinfection on the dynamics of microparasites. Integrative and Comparative selection using nested models. Theoretical Population Biology 69:145-53.

Gray, S., A. Power, D. Smith, A. J. Seaman, and N. S. Altman. 1991. Aphid transmission of barley yellow dwarf virus: Acquisition access periods and virus concentration requirements. Phytopathology 81:539-545. 
Halliday, F. W., J. Umbanhowar, and C. E. Mitchell. 2017. Interactions among symbionts operate across scales to influence parasite epidemics. Ecology Letters 20:1285-1294.

Handel, A., and P. Rohani. 2015. Crossing the scale from within-host infection dynamics to between-host transmission fitness: a discussion of current assumptions and knowledge. Philosophical Transactions of the Royal Society B 370:20140302.

Hoagland, D. R., and D. I. Arnon. 1938. The water culture method for growing plants without soil. California Agricultural Experiment Station Circular 347:32.

Katona, P., and J. Katona-Apte. 2008. The interaction between nutrition and infection. Clinical Infectious Diseases 46:1582-1588.

Lacroix, C., E. W. Seabloom, and E. T. Borer. 2014. Environmental nutrient supply alters prevalence and weakens competitive interactions among coinfecting viruses. The New Phytologist 204:424-433.

Lacroix, C., E. W. Seabloom, and E. T. Borer. 2017. Environmental nutrient supply directly alters plant traits but indirectly determines virus growth rate. Frontiers in Microbiology $8: 2116$.

Lange, B., M. Reuter, D. Ebert, K. Muylaert, and E. Decaestecker. 2014. Diet quality determines interspecific parasite interactions in host populations. Ecology and Evolution 4:3093-3102.

Liu, Y., H. Zhai, K. Zhao, B. Wu, and X. Wang. 2012. Two suppressors of RNA silencing encoded by cereal-infecting members of the family Luteoviridae. Journal of General Virology 93:1825-1830.

Mackay, I. M., K. E. Arden, and A. Nitsche. 2002. Real-time PCR in virology. Nucleic acids research 30:1292-305.

Maestre, F. T., and J. Cortina. 2004. Do positive interactions increase with abiotic stress? A test 
from a semi-arid steppe. Proceedings: Biological Sciences 271:179-182.

453 McCallum, H., A. Fenton, P. J. Hudson, B. Lee, B. Levick, R. Norman, S. E. Perkins, M. Viney,

A. J. Wilson, and J. Lello. 2017. Breaking beta: Deconstructing the parasite transmission

Mideo, N., S. Alizon, and T. Day. 2008. Linking within- and between-host dynamics in the evolutionary epidemiology of infectious diseases. Trends in Ecology \& Evolution 23:511-7.

Miller, T. E., J. H. Burns, P. Munguia, E. L. Walters, J. M. Kneitel, P. M. Richards, N. Mouquet, and H. L. Buckley. 2005. A critical review of twenty years' use of the resource-ratio theory.

Moreno, A. B., and J. J. López-Moya. 2020. When viruses play team sports: Mixed infections in

Nowak, H., and E. Komor. 2010. How aphids decide what is good for them: experiments to test aphid feeding behaviour on Tanacetum vulgare (L.) using different nitrogen regimes.

Pedersen, A. B., and A. Fenton. 2007. Emphasizing the ecology in parasite community ecology. Oecologia 163:973-984. community ecology of barley/cereal yellow dwarf viruses in Western US grasslands. Virus

471 R Core Team. 2018. R: A language and environment for statistical computing. R Foundation for Statistical Computing, Vienna, Austria.

Rochow, W., I. Muller, and F. Gildow. 1983. Interference between two luteoviruses in an aphid: lack of reciprocal competition. Phytopathology 73:919-922. 
475 de Roode, J. C., R. Culleton, S. J. Cheesman, R. Carter, and A. F. Read. 2004. Host

476 heterogeneity is a determinant of competitive exclusion or coexistence in genetically

477 diverse malaria infections. Proceedings of the Royal Society B 271:1073-1080.

478 Seabloom, E. W., C. D. Benfield, E. T. Borer, A. G. Stanley, T. N. Kaye, and P. W. Dunwiddie.

479 2011. Provenance, life span, and phylogeny do not affect grass species' responses to

$480 \quad$ nitrogen and phosphorus. Ecological Applications 21:2129-2142.

481 Seabloom, E. W., E. T. Borer, K. Gross, A. E. Kendig, C. E. Mitchell, E. A. Mordecai, and A. G.

482 Power. 2015. The community ecology of pathogens: coinfection, coexistence and community composition. Ecology Letters 18:401-415.

484 Seabloom, E. W., E. T. Borer, C. Lacroix, C. E. Mitchell, and A. G. Power. 2013. Richness and 485 composition of niche-assembled viral pathogen communities. PLoS ONE 8:e55675.

486 Smith, V. H., and R. D. Holt. 1996. Resource competition and within-host disease dynamics.

487 Trends in Ecology \& Evolution 11:386-389.

488 Smith, V., T. Jones, and M. Smith. 2005. Host nutrition and infectious disease: an ecological 489 view. Frontiers in Ecology and the Environment 3:268-274.

490 Soetaert, K., T. Petzoldt, and R. W. Setzer. 2010. Solving differential equations in R: Package 491 deSolve. Journal of Statistical Software 33:1-25.

492 Srinivasan, R., and J. M. Alvarez. 2007. Effect of mixed viral infections (potato virus Y-potato 493 leafroll virus) on biology and preference of vectors Myzus persicae and Macrosiphum 494 euphorbiae (Hemiptera: Aphididae). Journal of Economic Entomology 100:646-655.

495 Strauss, A. T., L. G. Shoemaker, E. W. Seabloom, and E. T. Borer. 2019. Cross-scale dynamics 496 in community and disease ecology: relative timescales shape the community ecology of 497 pathogens. Ecology:e02836. 
Susi, H., B. Barrès, P. F. Vale, and A. L. Laine. 2015a. Co-infection alters population dynamics of infectious disease. Nature Communications 6:1-8.

Susi, H., P. F. Vale, and A. L. Laine. 2015b. Host genotype and coinfection modify the relationship of within and between host transmission. American Naturalist 186:252-263.

Tilman, D. 1977. Resource competition between plankton algae: An experimental and theoretical approach. Ecology 58:338-348.

Tollenaere, C., H. Susi, and A.-L. Laine. 2016. Evolutionary and epidemiological implications of multiple infection in plants. Trends in Plant Science 21:80-90.

Vasco, D. A., H. J. Wearing, and P. Rohani. 2007. Tracking the dynamics of pathogen interactions: modeling ecological and immune-mediated processes in a two-pathogen

Wale, N., D. G. Sim, M. J. Jones, R. Salathe, T. Day, and A. F. Read. 2017. Resource limitation prevents the emergence of drug resistance by intensifying within-host competition.

Wen, F., and R. M. Lister. 1991. Heterologous encapsidation in mixed infections among four isolates of barley yellow dwarf virus. Journal of General Virology 72:2217-2223.

Whitaker, B. K., M. A. Rúa, and C. E. Mitchell. 2015. Viral pathogen production in a wild grass host driven by host growth and soil nitrogen. New Phytologist 207:760-768.

517 Wilkinson, T. L., R. Koga, and T. Fukatsu. 2007. Role of host nutrition in symbiont regulation:

518 Impact of dietary nitrogen on proliferation of obligate and facultative bacterial endosymbionts of the pea aphid Acyrthosiphon pisum. Applied and Environmental Microbiology 73:1362-1366. 
bioRxiv preprint doi: https://doi.org/10.1101/761254; this version posted April 16, 2020. The copyright holder for this preprint (which was not certified by peer review) is the author/funder, who has granted bioRxiv a license to display the preprint in perpetuity. It is made available under aCC-BY-NC-ND 4.0 International license.

521 Data availability: Data and code are publicly available on the Environmental Data Initiative

522 Data Portal: https://doi.org/10.6073/pasta/01e7bf593676a942f262623710acba13 
523 Table 1. Model estimates and 95\% credible intervals (CI) for statistical models of log-

524 transformed virus density.

\begin{tabular}{lcccc}
\hline Predictor & \multicolumn{2}{c}{ PAV } & \multicolumn{2}{c}{ RPV } \\
& Estimate & $95 \% \mathrm{CI}$ & Estimate & $95 \% \mathrm{CI}$ \\
\hline co-inoculation & $0.64^{*}$ & $0.06-1.22$ & 0.31 & $-0.36-0.97$ \\
N addition (N) & 0.17 & $-0.36-0.69$ & 0.03 & $-0.60-0.67$ \\
P addition (P) & -0.03 & $-0.54-0.47$ & 0.40 & $-0.29-1.10$ \\
co-inoculation:N & -0.61 & $-1.36-0.14$ & 0.33 & $-0.58-1.21$ \\
co-inoculation:P & -0.39 & $-1.12-0.35$ & -0.83 & $-1.82-0.14$ \\
N:P & -0.01 & $-0.72-0.70$ & 0.05 & $-0.91-0.98$ \\
co-inoculation:N:P & -0.04 & $-1.01-0.93$ & 0.51 & $-0.84-1.85$ \\
\hline
\end{tabular}

525 Note: Asterisk indicates estimate has 95\% CI that do not include zero, which suggests that "no

526 effect" is absent from the most probable estimate values. 
528 Table 2. Model estimates and 95\% credible intervals (CI) for statistical models of virus

529 transmission.

\begin{tabular}{|c|c|c|c|c|}
\hline \multirow[t]{2}{*}{ Predictor } & \multicolumn{2}{|c|}{ PAV } & \multicolumn{2}{|c|}{ RPV } \\
\hline & Estimate & $95 \% \mathrm{CI}$ & Estimate & $95 \% \mathrm{CI}$ \\
\hline density & 0.82 & $0.52-1.28$ & $1.30 *$ & $1.03-1.63$ \\
\hline co-infection & 0.68 & $0.14-2.99$ & 0.84 & $0.46-1.56$ \\
\hline $\mathrm{N}$ addition to source $\left(\mathrm{N}_{\text {source }}\right)$ & 1.72 & $0.72-4.15$ & 0.92 & $0.54-1.60$ \\
\hline $\mathrm{P}$ addition to source $\left(\mathrm{P}_{\text {source }}\right)$ & 0.83 & $0.35-1.91$ & 1.60 & $0.85-3.04$ \\
\hline $\mathrm{N}$ addition to recipient ( $\left.\mathrm{N}_{\text {recipient }}\right)$ & 0.75 & $0.20-2.83$ & $2.81 *$ & $1.25-6.69$ \\
\hline $\mathrm{P}$ addition to recipient ( $\left.\mathrm{P}_{\text {recipient }}\right)$ & $0.18^{*}$ & $0.05-0.57$ & 1.33 & $0.64-2.86$ \\
\hline $\mathrm{N}_{\text {source }}: \mathrm{P}_{\text {source }}$ & 0.79 & $0.24-2.53$ & 0.66 & $0.29-1.49$ \\
\hline $\mathrm{N}_{\text {recipient }}: \mathrm{P}_{\text {recipient }}$ & $8.88^{*}$ & $1.35-64.55$ & 0.9 & $0.26-3.12$ \\
\hline density: $\mathrm{N}_{\text {source }}$ & 2.01 & $0.95-5.13$ & 0.77 & $0.22-2.56$ \\
\hline density: $P_{\text {source }}$ & 2.72 & $0.84-10.55$ & 1.19 & $0.70-2.26$ \\
\hline density: $\mathrm{N}_{\text {recipient }}$ & 1.36 & $0.50-4.95$ & 0.78 & $0.47-1.38$ \\
\hline density: $P_{\text {recipient }}$ & $0.44^{*}$ & $0.17-0.95$ & 0.68 & $0.41-1.10$ \\
\hline co-infection: $\mathrm{N}_{\text {source }}$ & $0.22 *$ & $0.05-0.93$ & 1.87 & $0.90-3.85$ \\
\hline co-infection: $\mathrm{P}_{\text {source }}$ & 0.63 & $0.14-2.72$ & 0.82 & $0.37-1.79$ \\
\hline co-infection: $\mathrm{N}_{\text {recipient }}$ & 1.74 & $0.35-8.54$ & 0.87 & $0.29-2.60$ \\
\hline co-infection: $\mathrm{P}_{\text {recipient }}$ & $8.60^{*}$ & $2.00-41.27$ & 0.77 & $0.29-1.99$ \\
\hline density: $\mathrm{N}_{\text {source }}: \mathrm{P}_{\text {source }}$ & 0.13 & $0.01-1.50$ & 0.63 & $0.15-2.49$ \\
\hline density: $\mathrm{N}_{\text {recipient }}: \mathrm{P}_{\text {recipient }}$ & 7.24 & $0.88-110.44$ & $4.27 *$ & $1.57-15.61$ \\
\hline co-infection: $\mathrm{N}_{\text {source }}: \mathrm{P}_{\text {source }}$ & 1.71 & $0.27-11.02$ & 1.21 & $0.41-3.66$ \\
\hline co-infection: $\mathrm{N}_{\text {recipient }}: \mathrm{P}_{\text {recipient }}$ & 0.17 & $0.02-1.58$ & 0.81 & $0.16-4.16$ \\
\hline
\end{tabular}

530 Note: Asterisk indicates estimate (odds ratios) has $95 \%$ CI that do not include one, which

531 suggests that "no effect" is absent from the most probable estimate values. 


\section{Figure legends}

534 Figure 1. Diagram of the experimental design. (a) Source plants were grown in one of four

535 nutrient treatments and inoculated with one of three inoculation treatments. (b) Source plants

536 were harvested at eight different time points and tissue was cut into pieces. (c) Tissue was used

537 in molecular analysis to determine virus establishment and density. (d) Tissue was placed in

538 tubes with aphids, which were used to inoculate recipient plants and assess transmission.

539 Figure 2. The effects of nutrients and co-inoculation on ( $a, b)$ establishment (proportion of

540 source plants infected) and (e, f) log-transformed density (viruses per mg plant tissue) of (a, e)

541 PAV and (b, f) RPV over time (mean \pm 95\% nonparametric bootstrap confidence intervals).

542 Linear regression estimates of (c, g) PAV and (d, h) RPV (c, d) establishment and (g, h) log-

543 transformed density (mean $\pm 95 \%$ credible intervals).

544 Figure 3. The effects of source plant nutrition and infection status on (a) PAV and (b) RPV

545 transmission (proportion of recipient plants infected) over time, averaged over recipient plant

546 nutrient treatments (mean $\pm 95 \%$ nonparametric bootstrap confidence intervals; see Appendix

547 S2: Fig. S2 for averages over source plants). Regression relationships between transmission and

548 log-transformed virus density for (c) PAV (d) RPV. Vertical lines indicate overall average log-

549 transformed density for each virus. Regression estimates of (e) PAV and (f) RPV transmission

550 (mean $\pm 95 \%$ credible intervals) were taken at the average virus density for each treatment.

551 Figure 4. The predicted change in prevalence of $(a-c)$ PAV and $(d-f)$ RPV due to the presence

552 of the other virus in simulated plant populations grown with low nutrients, $\mathrm{N}$ addition, $\mathrm{P}$

553 addition, or both nutrients. Initial host population sizes were $I_{P}=1$ (when PAV was present), $I_{R}$

$554=1$ (when RPV was present), $I_{C}=0, N=4,000$. Parameter values represent transmission that $(\mathrm{b}$,

555 e) depends on virus density, (c, f) is independent of virus density, or (a, d) both. 


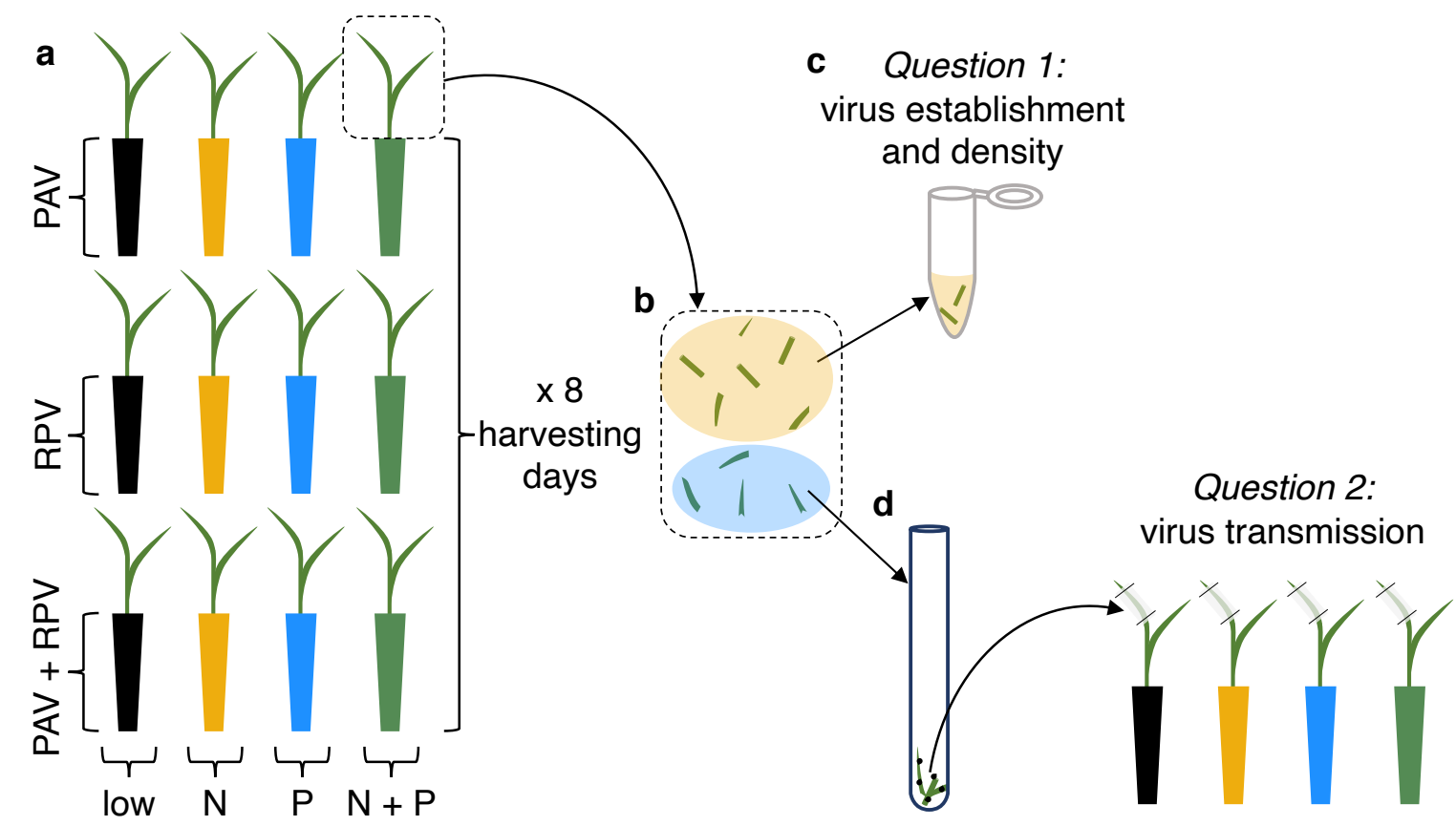


Figure 2
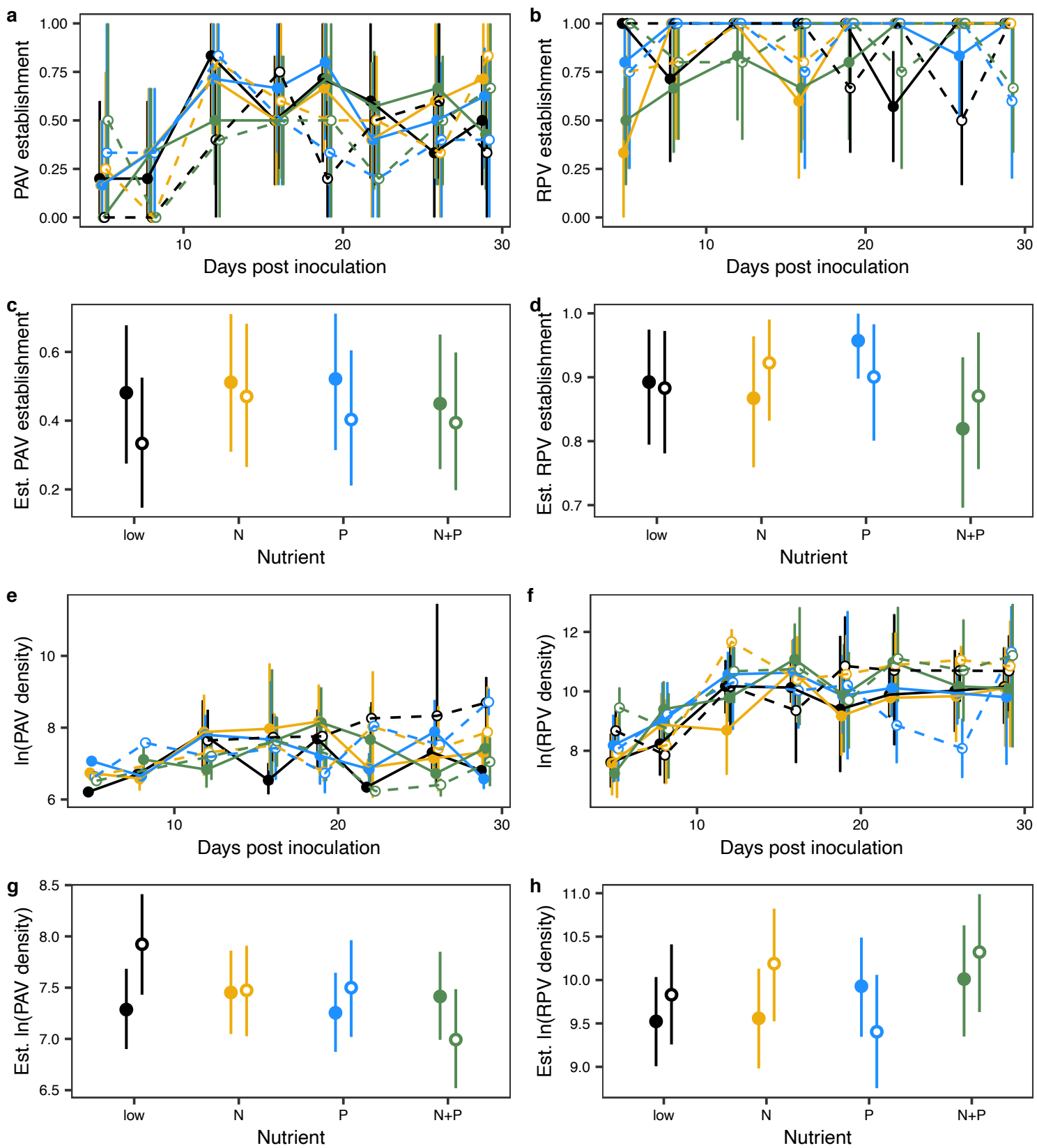
Figure 3
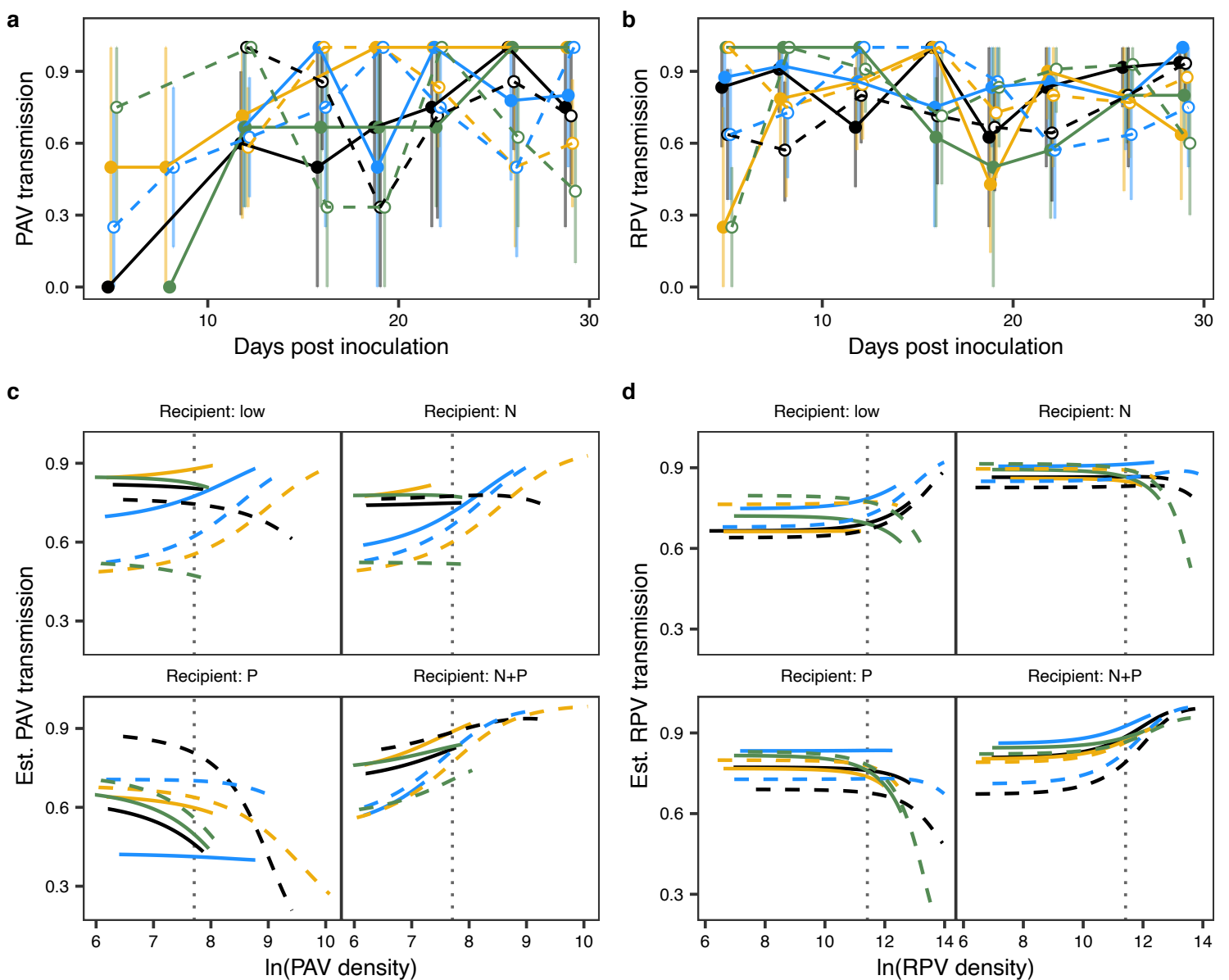

d
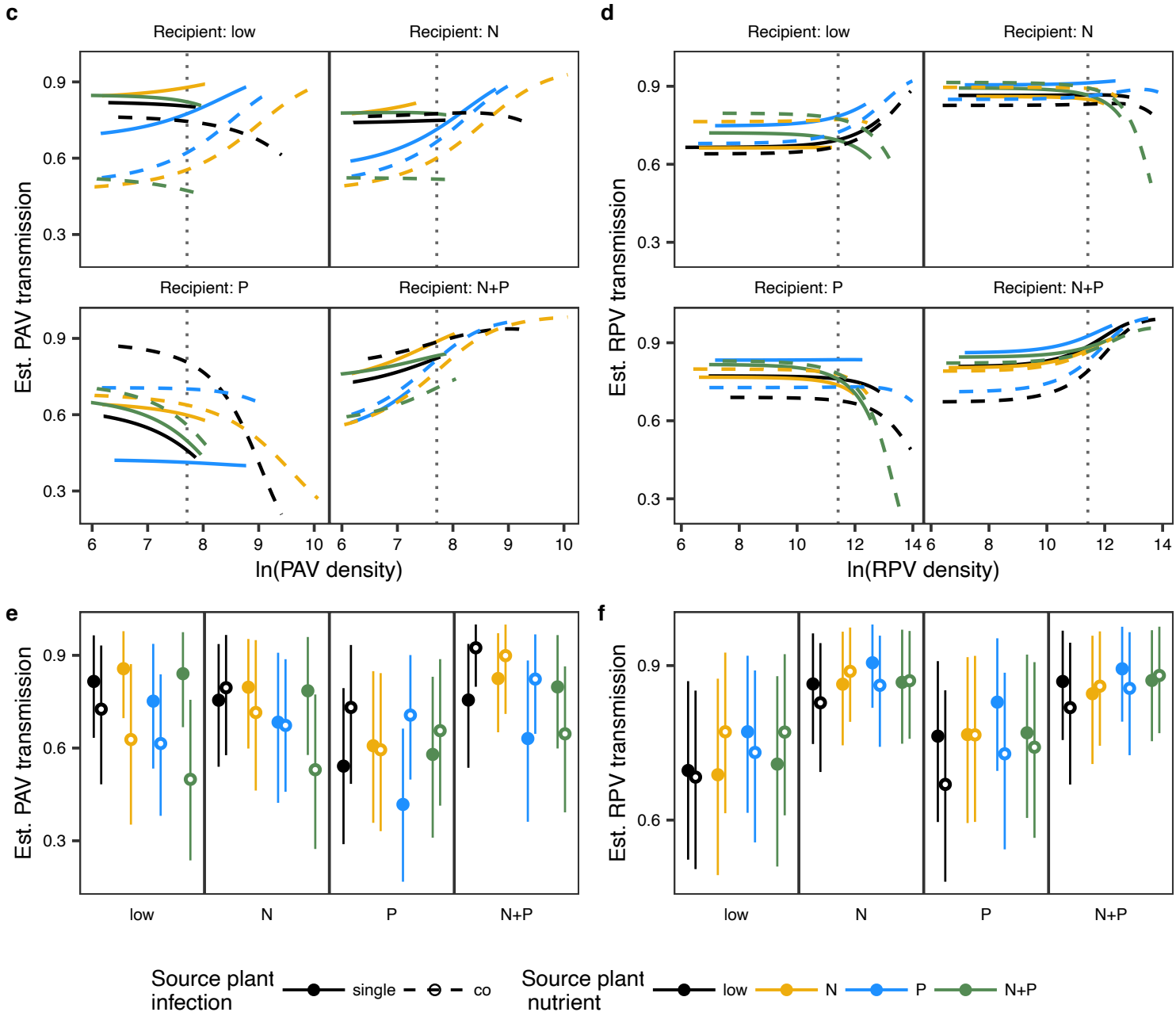


\section{$562 \quad$ Figure 4}

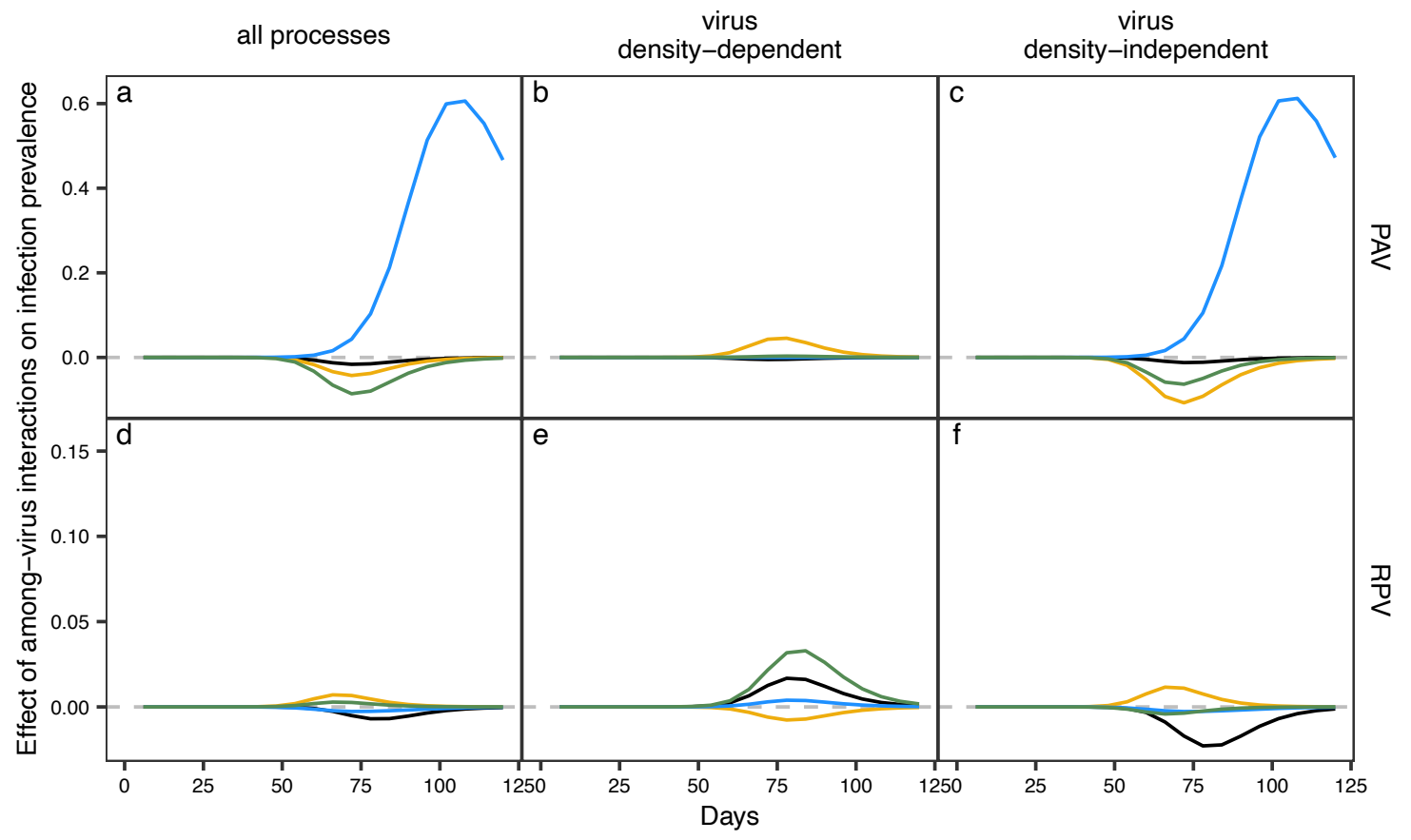

563

Nutrient $\longrightarrow$ low $\longrightarrow \mathrm{N} \longrightarrow \mathrm{P}=\mathrm{N}$ 